\title{
A Radial Velocity Dispersion Profile for the Fornax Dwarf Spheroidal Galaxy
}

\author{
Matthew G. Walker and Mario Mateo \\ Department of Astronomy, University of Michigan, 830 Dennison \\ Building, Ann Arbor, MI 48109-1090, USA
}

Edward W. Olszewski

Steward Observatory, University of Arizona, Tucson, AZ 85726, USA

Michael Woodroofe and Xiao Wang

Department of Statistics, University of Michigan, 439 West Hall, Ann Arbor, MI 48109-1092, USA

James Joyce

Department of Philosophy, University of Michigan, 435 South State St., Ann Arbor, MI 48109-1003, USA

\begin{abstract}
We have measured precise $( \pm 3 \mathrm{~km} / \mathrm{s})$ radial velocities for 180 stars in the Fornax dwarf spheroidal galaxy, spanning the region $\mathrm{R}=0$ to just beyond the nominal tidal radius. We perform a "classical" analysis of the resulting velocity dispersion profile. A mass-follows-light King model is ruled out, while a constant velocity dispersion model remains a good fit out to the limits of our dataset. For the constant velocity dispersion case, we calculate a velocity dispersion of $11.1 \pm 0.7 \mathrm{~km} / \mathrm{s}$, which implies a central $\mathrm{M} / \mathrm{L} /[\mathrm{M} / \mathrm{L}] \odot$ ratio of $7.6 \pm 1.0$.
\end{abstract}

Of 180 stellar velocities we have measured in the region of the Fornax dwarf spheroidal (dSph) galaxy, we take 160 to belong to members of Fornax. Figure 1 is the resulting velocity dispersion profile for Fornax, calculated using maximum likelihood statistics.

Over-plotted in Figure 1 are the best-fitting King (King 1966, solid curve) and constant velocity dispersion (dotted line) models. For the King model, which assumes mass follows light, we adopt the King radius $r_{0}=13.7 \pm 1.2$ arcmin, and tidal radius $\mathrm{r}_{t}=71.1 \pm 4.0$ arcmin, of Irwin \& Hatzidimitriou (1995). From these values, we find that the best-fit King profile yields a central velocity dispersion of $12.3 \mathrm{~km} / \mathrm{s}$, a value that implies a central M/L of $9 \pm 1$ in solar units (using the formalism of Richstone \& Tremaine 1986). However, the data do not show the King model's predicted drop-off in velocity dispersion with radius, hinting at a large dark matter component, and the best-fit King model is ruled out at greater than a 99 percent confidence level.

For the constant velocity dispersion model, we adopt the velocity dispersion derived from the entire 160 -star sample, $\sigma_{0}=11.1 \pm 0.7 \mathrm{~km} / \mathrm{s}$. This gives $\mathrm{M} / \mathrm{L}$ $=7 \pm 1$ in solar units. Though a constant velocity dispersion model becomes 


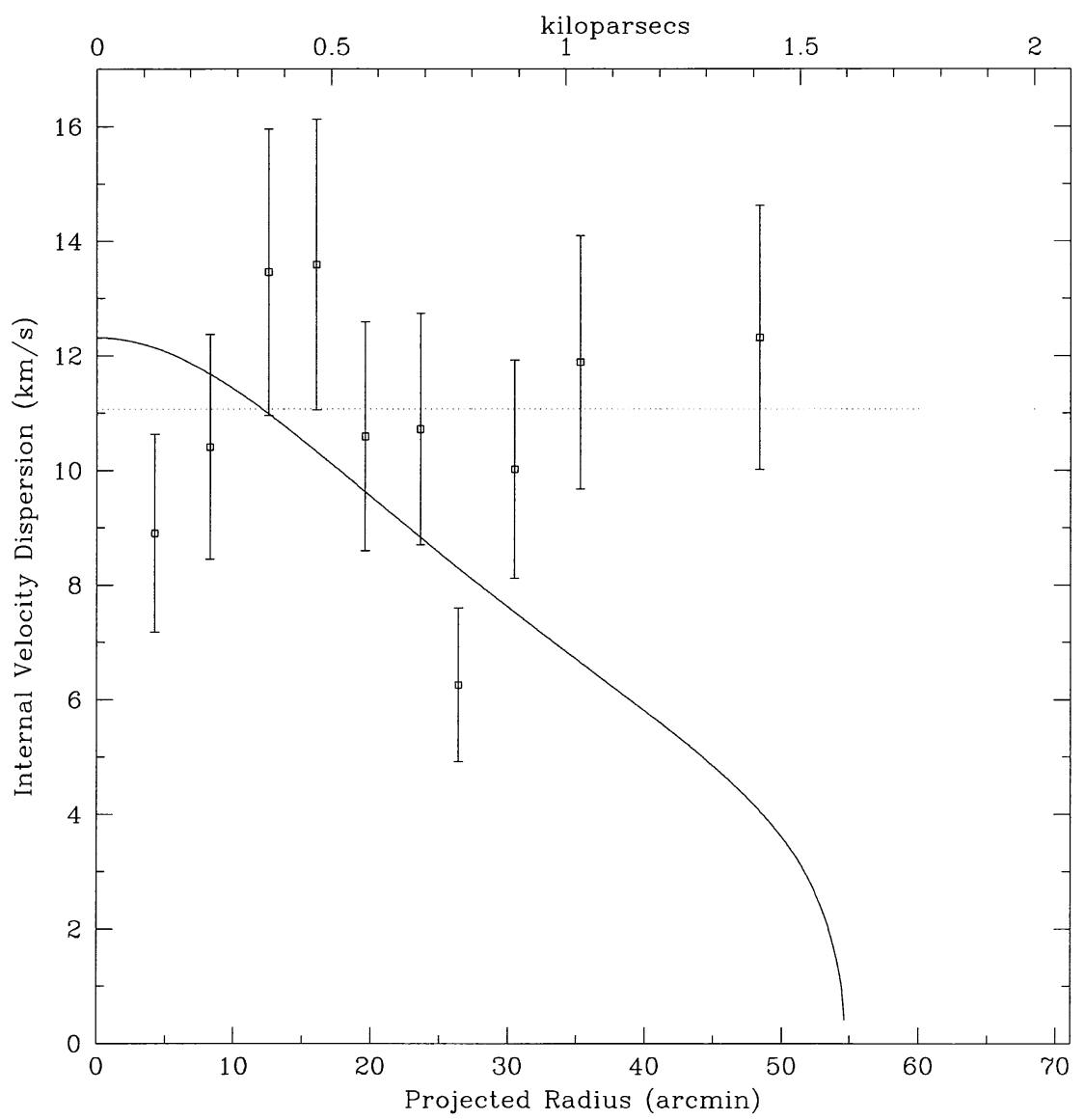

Figure 1. Measured Velocity Dispersion Profile for Fornax: The solid curve is the best-fitting King model; dashed line is the best-fitting constant dispersion.

unphysical at large radii, as it implies $\mathrm{M} \simeq 2 \sigma^{2} \mathrm{R} / \mathrm{G}$, we see relatively good agreement out to the limits of our sample. Thus, the radial extent to which the constant velocity model remains a good fit is unclear at this time, an uncertainty we plan to resolve using the Michigan MIKE Fiber System (MMFS), which saw first light in June 2003 at the Magellan telescopes.

\section{References}

Aaronson M. 1983, ApJ, 266, L11

Irwin M., Hatzidimitriou D. 1995, MNRAS, 277, 1354

King I.R. 1966, AJ, 71, 64

Richstone D.O., Tremaine S. 1986, AJ, 92, 72

Wilkinson M.I., Kleyna N.W., Evans N.W., Gilmore G. 2002, MNRAS, 330, 778 